\title{
CEP55 overexpression predicts poor prognosis in patients with locally advanced esophageal squamous cell carcinoma
}

\author{
WENPENG JIANG, ZHOU WANG and YANG JIA \\ Department of Thoracic Surgery, Shandong Provincial Hospital Affiliated to Shandong University, \\ Jinan, Shandong 250021, P.R. China
}

Received June 25, 2015; Accepted October 27, 2016

DOI: $10.3892 / \mathrm{ol} .2016 .5414$

\begin{abstract}
Development of esophageal squamous cell carcinoma (ESCC) involves alterations in multiple genes with corresponding proteins. Recent studies have demonstrated that centrosomal protein 55 (CEP55) shares certain features with oncogenes, and CEP55 overexpression is associated with the development and progression of malignant tumors. The present study aimed to analyze, for the first time, whether CEP55 expression is related to clinicopothalogic features in the esophageal squamous cell carcinoma (ESCC), as well as patient survival. A total of 110 patients with mid-thoracic ESCC who suffered from Ivor-Lewis were enrolled. The CEP55 expression profile of these patients in tumour tissues and corresponding healthy esophageal mucosa (CHEM) was detected by immunohistochemistry and semi-quantitative reverse transcription-polymerase chain reaction analyses. Correlations between CEP55 expression and clinicopathological factors were analyzed using $\chi^{2}$ test. The log-rank test was employed to calculate survival rate. A Cox regression multivariate analysis was performed to determine independent prognostic factors. The results demonstrated that CEP55 expression in ESCC was significantly higher than that of CHEM $(\mathrm{P}<0.001)$. Overexpression of CEP55 was significantly associated with differentiation degree $(\mathrm{P}=0.022)$, $\mathrm{T}$ stage $(\mathrm{P}=0.019)$, lymph node metastasis $(\mathrm{P}=0.033)$, clinicopathological staging $(\mathrm{P}=0.002)$ and tumor recurrence $(\mathrm{P}=0.021)$ in locally advanced ESCC patients. In addition, CEP55 overexpression was significantly associated with reduced overall survival of patients after surgery $(\mathrm{P}=0.012)$. The 5-year survival rate of patients without CEP55 overexpression was significantly higher than that of patients with CEP55 overexpression $(\mathrm{P}=0.012)$. Therefore, these
\end{abstract}

Correspondence to: Professor Zhou Wang, Department of Thoracic Surgery, Shandong Provincial Hospital Affiliated to Shandong University, 324 Jingwu Road, Jinan, Shandong 250021 P.R. China

E-mail: wangzhou620226@163.com

Key words: CEP55 overexpression, locally advanced esophageal squamous cell carcinoma, poor prognosis findings suggest that CEP55 overexpression correlates with poor prognosis in locally advanced ESCC patients.

\section{Introduction}

Esophageal carcinoma, which is the eighth leading cause of cancer-related deaths worldwide (1), occurs with great variation along with geography, ethnicity, and sociocultures. Esophageal squamous cell carcinoma (ESCC) is the most common pathological type in some East Asia countries, such as China and Japan, whereas adenocarcinoma occurs more commonly in Europe and the USA (2). To date, Ivor-Lewis esophagectomy has been chosen as the most valid means of resectable ESCC, as the majority of ESCC tend to occur in the middle thoracic esophagus. However, the benefits of radical resection through Ivor-Lewis esophagectomy remain unsatisfactory. Although the local tumor is completely removed via this method, $>50 \%$ of patients experience lymphatic metastatic recurrence in the 2 or 3 years after surgery (3), which is the main recurrence type.

As is well-known, the prognosis of patients with ESCC is TNM staging-specific. However, TNM staging is not accurate and sensitive enough to predict the prognosis of ESCC patients $(4,5)$. Therefore, it is necessary to investigate biological markers to predict the recurrence and prognosis of these patients. These markers may help to detect candidates with a high recurrence risk for postoperative adjuvant therapy. In our previous study, some molecular indicators (including C-C chemokine receptor type 7 and vascular endothelial growth factor-C) were identified that may be useful to predict lymphatic metastatic recurrence in $\mathrm{pN} 0$ esophageal squamous cell carcinoma (6). However, there has been no report on the correlation between the overexpression of centrosomal protein 55 (CEP55) and the prognosis of patients with ESCC after Ivor-Lewis esophagectomy.

CEP55, which has as important role in maintaining the proper function of the midbody structure, is the latest mitotic phosphoprotein to be found. CEP55 has an important role in the final stage of cell division, which involves the physical separation of the two daughter cells (7-9). Overexpression of CEP55 leads to cytokinesis defects and multinucleated cells increase, which may cause tumorigenesis. CEP55 overexpression has been found in various human tumors (10-14) and tumor cell lines (15). Overexpression of CEP55 in mammalian 
cells correlates with increased cell migration and invasion (16). Moreover, suppression of CEP55 expression predominantly impedes the growth of cancer cells, which is associated with increased apoptosis (10). The findings of these previous studies suggest that overexpression of CEP55 acts as a factor that contributes to poor patient prognosis in malignancies. However, to the best of our knowledge, there have been no reports that have established a correlation between the overexpression of CEP55 and the prognosis of ESCC. Therefore, the present study was designed to investigate the correlation between CEP55 overexpression in cancer tissues and prognosis in patients with ESCC after Ivor-Lewis esophagectomy.

\section{Materials and methods}

Ethics statement. The study protocol was approved by the Research Ethic Committee of Provincial Hospital Affiliated to Shandong University (Jinan, China). All patients and their relatives provided informed consent.

Patients and materials. Between March 2007 and May 2008, all of the patients enrolled in the present study $(n=110)$ suffered from mid-thoracic ESCC and had undergone Ivor-Lewis esophagectomy with two-field lymph node dissection in our department, and were retrospectively studied. The inclusion criteria were as follows: i) Mid-thoracic esophageal squamous cell carcinoma pathologically diagnosed as postoperative pathological stage $\mathrm{T} 2-4 \mathrm{aN} 0-2 \mathrm{M} 0$; ii) no preoperative radiotherapy or chemotherapy; iii) without surgical contraindication; iv) metastasis of cervical or supra-clavicular lymph node was excluded prior to surgery; v) All of the operations were radical resections identified by the postoperative pathology; vi) there was no serious operative complication; and vii) number of lymph nodes dissected was $>12$. The study group consisted of 79 men and 31 women aged 43-72 years. Pathological staging complied with the classification guidelines outlined by the International Union Against Cancer in 2009 (17). Patients were routinely followed-up during the first six years following surgery. Data of recurrent disease were recorded in completion. Clinicopathological characteristics of the 110 patients are listed in Table I.

Each specimen from the 110 patients was cut into two sections. An esophageal cancer specimen of at least $0.5 \times 0.5 \times 0.5 \mathrm{~cm}$ in size was used for RNA extracting. Each esophageal cancer tissue was labeled, wrapped quickly in foil, snap-frozen in liquid nitrogen for $1 \mathrm{~min}$, and stored at $-80^{\circ} \mathrm{C}$ until subsequent RNA extraction. The remaining esophageal cancer tissue specimen was fixed in a $10 \%$ formaldehyde solution for histopathological examination. Histological examination confirmed that all the cancer tissues studied were squamous cell carcinomas.

CHEM samples were harvested for each patient from a sample site located $>5 \mathrm{~cm}$ from the margin of ESCC. All of the CHEM samples from the 110 patients were analyzed for CEP55 expression via immunohistochemical and semi-quantitative reverse transcription-polymerase chain reaction (RT-PCR) analysis.

Immunohistochemistry. Immunohistochemical analysis, using the streptavidin-peroxidase (SP) method, was performed to
Table I. CEP55 expression and clinicopathological characteristics in patients with esophageal squamous cell carcinoma.

\begin{tabular}{|c|c|c|c|c|}
\hline \multirow[b]{2}{*}{ Characteristic } & \multirow[b]{2}{*}{ Patients } & \multicolumn{2}{|c|}{$\begin{array}{c}\text { CEP55 } \\
\text { expression }\end{array}$} & \multirow[b]{2}{*}{ P-value } \\
\hline & & $(-)$ & $(+)$ & \\
\hline Total & 110 & 47 & 63 & \\
\hline Gender & & & & 0.336 \\
\hline Male & 79 & 36 & 43 & \\
\hline Female & 31 & 11 & 20 & \\
\hline Age (years) & & & & 0.392 \\
\hline$<60$ & 52 & 20 & 32 & \\
\hline$\geqq 60$ & 58 & 27 & 31 & \\
\hline Tumor length $(\mathrm{cm})$ & & & & 0.06 \\
\hline$<5$ & 45 & 24 & 21 & \\
\hline$\geqq 5$ & 65 & 23 & 42 & \\
\hline Differentiation & & & & 0.022 \\
\hline Well-moderate & 71 & 36 & 35 & \\
\hline Poor & 39 & 11 & 28 & \\
\hline $\mathrm{pT}$ & & & & 0.019 \\
\hline $\mathrm{T} 2$ & 38 & 22 & 16 & \\
\hline $\mathrm{T} 3$ & 72 & 25 & 47 & \\
\hline $\mathrm{pN}$ & & & & 0.033 \\
\hline $\mathrm{N} 2$ & 24 & 6 & 18 & \\
\hline N1 & 52 & 21 & 31 & \\
\hline NO & 34 & 20 & 14 & \\
\hline TNM stage & & & & 0.002 \\
\hline II & 36 & 23 & 13 & \\
\hline III & 74 & 24 & 50 & \\
\hline Recurrence & & & & 0.021 \\
\hline Yes & 49 & 15 & 34 & \\
\hline No & 61 & 32 & 29 & \\
\hline
\end{tabular}

detect the levels of CEP55 expression in each tissue specimen. Slides were stained according to the manufacturer's protocols for CEP55 (Beijing Biosynthesis Biotechnology Co., Ltd., Beijing, China). Formalin-fixed paraffin-embedded sections $(4 \mu \mathrm{m})$ were heated at $65^{\circ} \mathrm{C}$ for $30 \mathrm{~min}$ and were dewaxed in xylene, rehydrated through a graded alcohol series and placed in an endogenous peroxide block for $10 \mathrm{~min}$. Antigen retrieval was performed using $10 \mathrm{mM}$ of citrate buffer in a microwave for $15 \mathrm{~min}$. Tissue sections were subsequently incubated at $4^{\circ} \mathrm{C}$ overnight with anti-CEP55 rabbit polyclonal antibody (bs-7742R; Beijing Biosynthesis Biotechnology Co., Ltd.) at a dilution of 1:150 in PBS. Secondary processing of the tissue samples was performed with an SP kit and a universal secondary antibody kit (SP-9001) according to the manufacturer's instructions (Beijing Zhongshan Golden Bridge Biotechnology Co., Ltd., Beijing, China). Briefly, following incubation overnight with the primary antibody, the secondary biotinylated antibody and subsequent avidin-biotin complex reagent were incubated for $30 \mathrm{~min}$ at $37^{\circ} \mathrm{C}$, respectively. The tissues were then rinsed with PBS three times (5 min each 

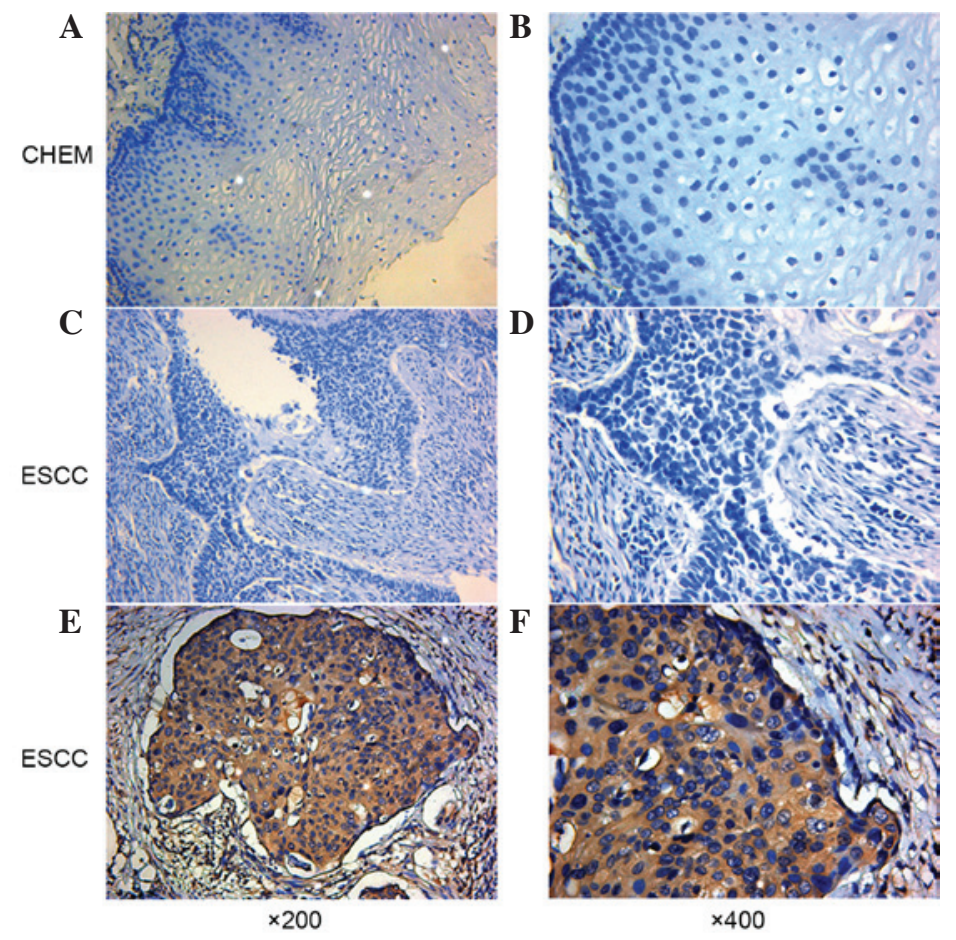

Figure 1. Immunohistochemical staining of CEP55 in ESCC and CHEM. (A and B) Representative negative expression of CEP55 in CHEM. (C and D) Representative low expression of CEP55 in ESCC. (E and F) Representative high expression of CEP55 in ESCC. Magnification, x200 and x400 (original magnification). Nuclei were counterstained with hematoxylin. CEP55, centrosomal protein 55; ESCC, esophageal squamous cell carcinoma; CHEM, corresponding healthy esophageal mucosa.

time). Staining was visualized using diaminobenzidine and tissues were counterstained with hematoxylin. As the negative control, the primary antibody was replaced with PBS.

The immunohistochemical score was calculated by combining the proportion score (percentage of positive stained cells) with the staining intensity score. Specimens were examined under a light microscope. In five randomly selected fields of vision per-section, positively stained cells among 100 cells were assessed and quantified (percentage). The mean percentage of the five fields was used to identify the proportion score in a six-category grading system $(0$, negative; $1,1-10 \%$; $2,11-25 \% ; 3,26-50 \% ; 4,51-75 \%$; and 5, $>75 \%$ ). Staining intensity was scored as follows: 0 , negative; 1 , weak; 2 , moderate; and 3, strong. Both of the scores were multiplied to form the immunohistochemical score. All sections were examined by two independent pathologists who were blinded to the clinical data.

Receiver operating characteristics (ROC) curve. The cut-off score for CEP55 overexpression was screened based on the ROC curve. Raw data of CEP55 expression levels in the ESCC and CHEM groups were analyzed using a MedCalc v13.0.2.0 statistical software package (MedCalc Software bvba, Ostend, Belgium). The score closest to the point of both maximum sensitivity and specificity was selected as the cut-off score.

Semi-quantitative RT-PCR analysis. Total RNA of each specimen was extracted using a TRIzol (Invitrogen; Thermo Fisher Scientific, Inc. Waltham, MA, USA) one-step procedure according to the protocol provided by the manufacturer.
RNA purity and concentration were determined by a standard ultraviolet spectrophotometric assay. RT-PCR was performed using PrimeScript RT reagent kit (Takara Biotechnology Co., Ltd., Dalian, China) according to the manufacturer's protocol. To remove genomic DNA, total RNA was mixed with $2.0 \mu \mathrm{l}$ 5X gDNA Eraser Buffer and $1.0 \mu \mathrm{l}$ gDNA Eraser. RNase free water was then added to the mixture to make a total reaction volume of $10 \mu \mathrm{l}$. The thermal profile was $42^{\circ} \mathrm{C}$ for $2 \mathrm{~min}$ followed by $4^{\circ} \mathrm{C}$, at which point the fluid was ready for the next step. In the RT step, the reaction was performed in a total volume of $25 \mu \mathrm{l}$, containing $4 \mu \mathrm{l}$ of $5 \mathrm{X}$ PrimeScript Buffer 2, $1 \mu 1$ PrimeScript RT Enzyme Mix1, $1 \mu 1$ RT Primer Mix, $10 \mu \mathrm{l}$ RNase Free $\mathrm{dH}_{2} \mathrm{O}$ and $10 \mu \mathrm{l}$ of the aforementioned fluid. The thermal profile was as follows: $37^{\circ} \mathrm{C}$ for $15 \mathrm{~min}, 85^{\circ} \mathrm{C}$ for $5 \mathrm{sec}$, followed by $4^{\circ} \mathrm{C}$. $\beta$-actin was used as an internal control. Primers were designed according to previous reports (16) as follows: CEP55, 5'-end primer 5'-TTGGAACAACAGATG CAGGC-3' and 3'-end primer 5'-GAGTGCAGCAGTGGG ACTTT-3'; and $\beta$-actin, 5'-end primer 5'-AGAGCCTCGCCT TTGCCGATCC-3' and 3'-end primer 5'-CTGGGCCTCGTC GTCGCCCACATA-3'.

Following an initial denaturation at $94^{\circ} \mathrm{C}$ for $5 \mathrm{~min}$, samples were amplified by 35 cycles of $94^{\circ} \mathrm{C}$ for $30 \mathrm{sec}$, $58^{\circ} \mathrm{C}$ for $30 \mathrm{sec}, 72^{\circ} \mathrm{C}$ for $30 \mathrm{sec}$ and final extension at $72^{\circ} \mathrm{C}$ for $10 \mathrm{~min}$. PCR products were visualized by electrophoresis through $1 \%$ agarose gels stained with ethidium bromide. Gel images were obtained using an Alphalmager 2200 UV-image analyzer (Alpha Innotech, San Leandro, CA, USA). Ratios of CEP55/ $\beta$-actin were used to semi-quantify the CEP55 expression levels. PCR was repeated in triplicate for all samples and the data were analyzed using the comparative Cq method (18). 
Adjuvant therapies. To date in China, there have been no unanimous guidelines on postoperative adjuvant therapy after radical resection for the treatment of ESCC. Therefore, National Comprehensive Cancer Network (NCCN) guidelines are often used, which are not appropriate for the Chinese population with ESCC. In our department the indications for postoperative adjuvant treatment are on the basis of tumor stage, clinicians' preferences and patients' willingness for treatment or economic status. Typically, patients with pT3-4 are advised to receive radiotherapy and those with $\mathrm{pN} 1$ should receive chemotherapy as a minimum. In the patients enrolled in the present study, nine received postoperative radiotherapy alone. Postoperative chemotherapy was administered to 12 patients for $>4$ cycles, predominantly paclitaxel and cisplatin/carboplatin, and 64 patients received combined chemoradiotherapy.

Follow-up after surgery and diagnosis of recurrence. Patients were regularly reexamined every 3-6 months in the three years following surgery. After three years, follow-up took place every 6-12 months. These follow-up appointments included a thorough physical examination and chest and upper abdomen computed tomography scans. Positron emission tomography-computed tomography scanning was administered to specific patients if necessary. Examinations were compared with preoperative imaging data. If there was progressive lymph node enlargement, biopsy was the first choice to identify possible lymph node metastatic recurrence. Patients with mediastinal lymph node enlargement identified in CT scans were advised to undertake PET-CT examination if biopsy was difficult to achieve. A total of 18 patients were diagnosed with lymphatic metastatic recurrence by PET-CT. Some patients' metastases were diagnosed using biopsies. If new lesions were identified in other organs, the patient was clinically diagnosed with metastatic cancer after excluding the primary tumor. The study follow-up period ended in February 2014; the longest follow-up period was six years.

Statistical methods. The Mann-Whitney U test was used to identify differences in CEP55 expression after immunohistochemical analysis. The $\chi^{2}$ test was employed to analyze the correlations between CEP55 overexpression and clinicopathological factors. Univariate analysis was performed by modeling Kaplan-Meier survival curves. The log-rank test was used to calculate the survival rate. Multivariate analysis was performed using the Cox proportional hazard model. $\mathrm{P}<0.05$ was considered to indicate a statistically significant difference. Statistical data were obtained using an SPSS software package (SPSS 17.0; SPSS, Inc., Chicago, IL, USA).

\section{Results}

Expression of CEP55 in ESCC tissue and CHEM. Using immunohistochemical analysis, positive expression of CEP55 was detected as yellow or brownish yellow staining in the cytoplasm. Positive CEP55 staining was readily detected in ESCC, whereas negative or low staining was predominantly observed in CHEM (Fig. 1). Expression levels of CEP55 in ESCC were markedly increased, as compared with in CHEM (Fig. 2). According to the ROC curve (Fig. 3), the threshold value of 8 was the closest to the point with both maximum

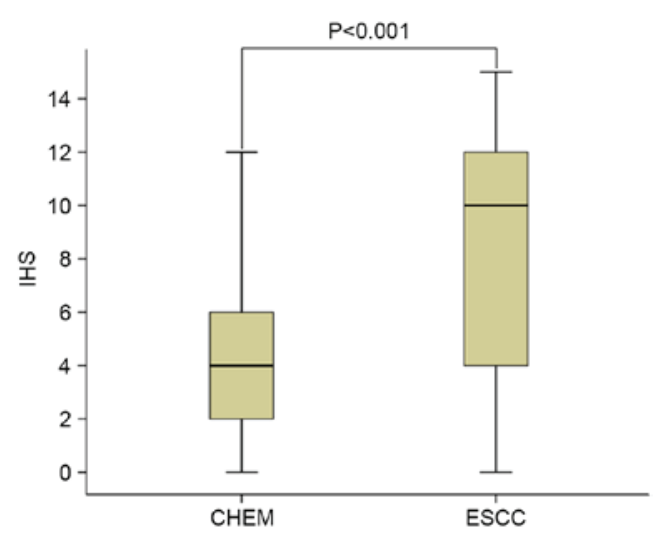

Figure 2. CEP55 expression in different tissues. IHS of CEP55 in CHEM and ESCC. Data are represented as a box plot and analyzed by Mann-Whitney U test. IHS, immunohistochemical scores; CEP55, centrosomal protein 55; CHEM, corresponding healthy esophageal mucosa; ESCC, esophageal squamous cell carcinoma.

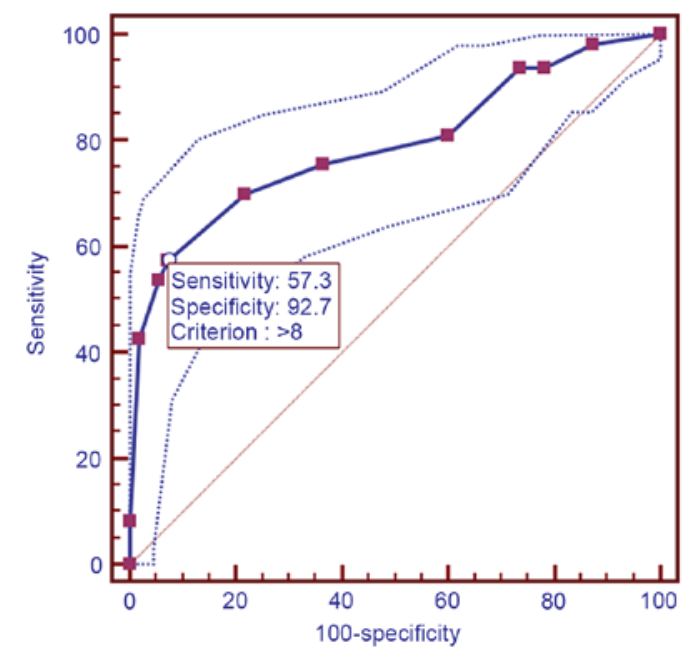

Figure 3. Receiver operating characteristics analysis of centrosomal protein 55 expression and the selection of cut-off score.

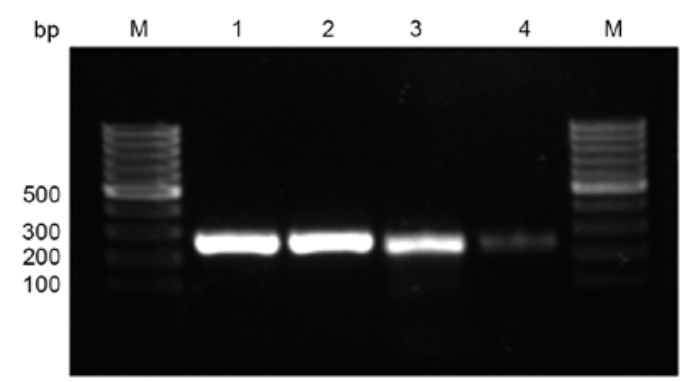

Figure 4. Expression of CEP55 mRNA, as detected by reverse transcription-quantitative polymerase chain reaction analysis. Lane 1, representative $\beta$-actin mRNA expression in ESCC; lane 2, representative $\beta$-actin mRNA expression in CHEM; lane 3, representative CEP55 mRNA expression in ESCC; and lane 4, representative CEP55 mRNA expression in CHEM. M, molecular marker; bp, basepair; IHS, immunohistochemical scores; CEP55, centrosomal protein 55; CHEM, corresponding healthy esophageal mucosa; ESCC, esophageal squamous cell carcinoma.

sensitivity $(57.3 \%)$ and specificity $(92.7 \%)$; thus, a score of 8 was selected as the cut-off value. The area under the curve was 
Table II. Cox regression analysis for risk factors of 5-year survival.

\begin{tabular}{lcccccc}
\hline Characteristic & $\mathrm{B}$ & SE & Wald & P-value & OR & $95.0 \%$ CI \\
\hline Gender & -0.265 & 0.268 & 0.981 & 0.322 & 0.767 & $0.454-1.297$ \\
Age & 0.291 & 0.241 & 1.455 & 0.228 & 1.338 & $0.834-2.148$ \\
Tumor size & 0.368 & 0.338 & 1.189 & 0.275 & 1.445 & $0.746-2.801$ \\
Lymph node metastasis & 2.429 & 0.356 & 46.484 & 0.000 & 11.348 & $5.645-22.813$ \\
Invasion depth & 1.015 & 0.479 & 4.493 & 0.034 & 2.760 & $1.079-7.056$ \\
TNM stage & 1.594 & 0.737 & 4.679 & 0.031 & 4.926 & $1.161-20.889$ \\
CEP55 overexpression & 0.041 & 0.244 & 0.028 & 0.867 & 1.042 & $0.646-1.680$ \\
Differentiation & 0.531 & 0.267 & 3.941 & 0.047 & 1.700 & $1.007-2.870$ \\
\hline
\end{tabular}

B, regression coefficient; SE, standard error; OR, odds ratio; CI, confidence interval.
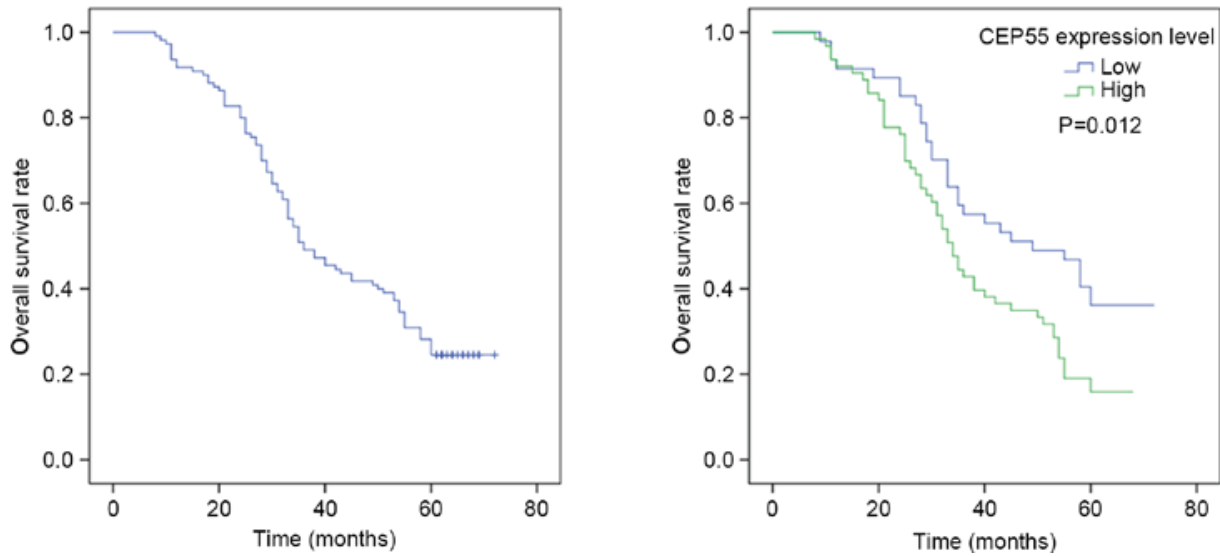

Figure 5. Kaplan-Meier analysis of overall survival in patients with ESCC. (A) Overall 5-year survival rate of 110 patients with ESCC. (B) The 5-year survival rate of ESCC patients with CEP55 overexpression vs. patients without CEP55 overexpression. CEP55, centrosomal protein 55; ESCC, esophageal squamous cell carcinoma.

0.79 [95\% confidence interval $(\mathrm{CI}): 0.730-0.842 ; \mathrm{P}<0.0001]$ Samples with a immunohistochemical score $>8$ were identified as exhibiting high CEP55 expression, whereas samples with scores $<8$ were defined as having low or negative CEP55 expression. To validate the results of immunohistochemical analysis, PCR was employed to analyze CEP55 mRNA expression levels in different tissues. The results were consistent with those determined through the immunohistochemical method (Fig. 4).

Correlation between CEP55 overexpression and clinical characteristics. CEP55 overexpression was identified in ESCC tissues from 63 patients. Diagnostic sensitivity was demonstrated to be $57.3 \%$ (63/110; Table I). The correlations between CEP55 expression and clinicopathological features are shown in Table I. $\chi^{2}$ analysis indicated that CEP55 overexpression was significantly associated with tumor differentiation degree $(\mathrm{P}=0.022)$, depth of invasion $(\mathrm{P}=0.019)$, lymph node metastasis $(\mathrm{P}=0.033)$, TNM stage $(\mathrm{P}=0.002)$ and tumor recurrence $(\mathrm{P}=0.021$; Table I). No other clinicopathological parameter was found to be associated with CEP55 overexpression (Table I). Notably, all the positive correlations with CEP55 overexpression were aggressive clinicopathological features of patients with ESCC.
Correlation between CEP55 expression and prognosis. The 1-, 3-, and 5-year overall survival rates of 110 patients were $91.8,49.8$ and $30.4 \%$, respectively. The median survival time was 36.0 months (95\% CI: 27.4-44.6 months). Univariate analysis indicated that CEP55 expression levels $(\mathrm{P}=0.012)$ were significant prognostic factors. The 5-year survival rate of patients without CEP55 overexpression in ESCC tissues was significantly higher than that of patients with CEP55 overexpression (Fig. 5). The results of Cox regression multivariate analysis revealed that $\mathrm{pT}$ status, lymph node metastasis, TNM stage and tumor differentiation degree were independent relevant factors (Table II).

\section{Discussion}

Esophageal cancer is a common lethal malignancy that typically derives from esophageal mucosa. As a predominant pathological type, ESCC has a higher incidence rate than adenocarcinoma in China (2). As a high-grade malignancy, the prognosis of ESCC is far from satisfactory despite great advances in the compressive therapy of ESCC. Even following surgery, patients suffer from an overall 5-year survival rate of 30-50\% (19). Despite radical resection, $>50 \%$ of patients relapse in the 2 or 3 years following surgery. The most common recurrence type is lymphatic 
metastatic recurrence (2). The distinctive embryological structure of the esophagus is characterized by the presence of lymph-vessels in muscularis mucosae. The process of lymphatic drainage of the esophagus is complex with a rich lymphatic network. Lymph node metastasis may present as regional metastasis, skipping metastasis, or distant metastasis (20-22). Lymph node metastasis of esophageal cancer typically occurs when the primary tumor is particularly small.

It is widely accepted that complete resection is the preferred option to cure resectable mid-thoracic ESCC. On the basis of oncology, subtotal oesophagectomy accompanied with three-field lymphadenectomy (McKeown procedure) is regarded as the optimal approach for mid-thoracic ESCC. However, this procedure is highly invasive and was accompanied by a high incidence of complications, such as recurrent nerve paralysis (23-26). Through three-field lymph node dissection, only $20-30 \%$ of patients with ESCC have been identified as having cervical or supraclavicular lymphatic metastasis (27). For the majority of patients with ESCC, three-field lymph node dissection is used for accurate staging rather than an improvement in long-term survival. Therefore, whether three-field lymph node dissection should be performed in all patients with thoracic ESCC remains controversial, and this procedure has not been widely adopted in clinics. Modified Ivor-Lewis esophagectomy via a thoracoabdominal two-field lymph node dissection is preferable to treat ESCC, as previously reported (28), due to its predilection for the middle thoracic esophagus. The disadvantage of this procedure is that cervical lymph node dissection cannot be accomplished simultaneously. However, some patients identified as pN0 stage via postoperative pathology actually suffer from cervical lymph node metastasis, as lymph node metastasis of ESCC is not detected. Therefore, this creates a problem of how ESCC patients with a probability of high lymphatic node metastasis can be identified. For patients who have a high probability of lymphatic node metastasis, cervical lymph node dissection can be performed simultaneously or postoperative radiotherapy can be utilized, which assesses the neck, supraclavicular region and superior mediastinum for lymphatic node metastasis.

To date in China, there is no consensus on whether an adjuvant therapy is required for ESCC patients with complete resection. Thus, the NCCN esophageal cancer guidelines are often referenced in the clinic. The NCCN treatment guidelines shows that patients with ESCC do not have to accept an adjuvant therapy after the complete removal of a tumor. However, considering the poor prognosis of ESCC patients after radical surgery, we propose that an adjuvant therapy is necessary for specific patients to improve their prognosis, and patients with ESCC should be treated individually. To achieve this aim, the invasiveness and the lymph node metastatic potency of the ESCC must be identified. Therefore, it is necessary to identify novel indicators to predict patient survival more accurately in clinical practice.

A human gene, CEP55, which is located in 10q23.33 encodes a protein capable of homodimerization. It was reported that CEP55 protein localizes to the centrosome in interphase cells and transfers to the midbody during cytokinesis. CEP55 has an essential role in membrane fission events $(29,30)$. In the final stage of the cell cycle, physical separation of the cytoplasmic volumes occurs and the two prospective daughter cells arise (31). During this stage, an intercellular bridge containing the midbody ring is formed (32). This ring regulates membrane fission and fusion events $(29,33)$. Failure of cytokinesis results in tetraploid cells, which are chromosomally unstable and hence more prone to tumorigenesis (8). Overexpression of CEP55 causes cytokinesis defects via an increase of chromosomally unstable binucleated cells, suggesting CEP55 overexpression is associated with tumorigenesis.

CEP55 overexpression is found in various cancer cell lines, while its expression is barely detected in normal tissues by expression-profile analyses using microarrays (34). As reported, CEP55 overexpression has been detected in hepatocarcinoma (11), colon carcinoma (34), oral cavity squamous cell carcinoma (13) and lung cancer (14). Notably, the present study is the first to report CEP55 overexpression in the majority of ESCC specimens examined.

The present study investigated the correlation between CEP55 expression in cancer tissues and the prognosis of patients with locally advanced ESCC after Ivor-Lewis esophagectomy. The 5-year survival rate of patients with CEP55 overexpression in tumor issue was significantly lower than that of patients without CEP55 overexpression. Furthermore, overexpression of CEP55 was demonstrated to be associated with the differentiation degree, T stage, lymph node metastasis, TNM staging and tumor recurrence in locally advanced ESCC patients.

In conclusion, CEP55 overexpression predicts poor prognosis and is associated with aggressive clinicopathological features in ESCC. This requires a decision on whether cervical lymph node dissection should be performed and which patients with ESCC should be selected to receive postoperative adjuvant therapy. As a tumour accelerator, CEP55 may be a novel therapeutic target for cancer therapy. Further research should be performed to identify whether knockdown of the CEP55 gene can retard the invasiveness of ESCC cells. The mechanism through which CEP55 regulates the growth of ESCC should be researched to confirm the potential effectiveness of CEP55 as a therapeutic target of ESCC in clinical practice.

\section{References}

1. Valverde CM, Macarulla T, Casado E, Ramos FJ, Martinelli E and Tabernero J: Novel targets in gastric and esophageal cancer. Crit Rev Oncol Hematol 59: 128-138, 2006.

2. Jemal A, Bray F, Center MM, Ferlay J, Ward E and Forman D: Global cancer statistics. CA Cancer J Clin 61: 69-90, 2011.

3. Nakagawa S, Kanda T, Kosugi S, Ohashi M, Suzuki T and Hatakeyama K: Recurrence pattern of squamous cell carcinoma of the thoracic esophagus after extended radical esophagectomy with three-field lymphadenectomy. J Am Coll Surg 198: 205-211, 2004.

4. D'Annoville T, D'Journo XB, Loundou A, Trousse D, Dahan L, Doddoli C, Seitz JF and Thomas PA: Prognostic impact of the extracapsular lymph node involvement on disease-free survival according to the 7th edition of American joint committee on cancer staging system. Eur J Cardiothorac Surg 44: e207-e211; discussion e211, 2013.

5. Hou X, Wei JC, Fu JH, Wang X, Zhang LJ, Lin P and Yang HX: Proposed modification of the seventh American joint committee on cancer staging system for esophageal squamous cell carcinoma in Chinese patients. Ann Surg Oncol 21: 337-342, 2014.

6. Song Y, Wang Z, Liu X, Jiang W and Shi M: CCR7 and VEGF-C: Molecular indicator of lymphatic metastatic recurrence in $\mathrm{pN} 0$ esophageal squamous cell carcinoma after Ivor-Lewis esophagectomy? Ann Surg Oncol 19: 3606-3612, 2012.

7. Fabbro M, Zhou BB, Takahashi M, Sarcevic B, Lal P, Graham ME, Gabrielli BG, Robinson PJ, Nigg EA, Ono Y and Khanna KK: Cdk1/Erk2- and Plk1-dependent phosphorylation of a centrosome protein, Cep55, is required for its recruitment to midbody and cytokinesis. Dev Cell 9: 477-488, 2005. 
8. Morita E, Sandrin V, Chung HY, Morham SG, Gygi SP, Rodesch CK and Sundquist WI: Human ESCRT and ALIX proteins interact with proteins of the midbody and function in cytokinesis. EMBO J 26: 4215-4227, 2007.

9. Zhao WM, Seki A and Fang G: Cep55, a microtubule-bundling protein, associates with centralspindlin to control the midbody integrity and cell abscission during cytokinesis. Mol Biol Cell 17: 3881-3896, 2006.

10. Sakai M, Shimokawa T, Kobayashi T, Matsushima S, Yamada Y, Nakamura Y and Furukawa Y: Elevated expression of C10orf3 (chromosome 10 open reading frame 3 ) is involved in the growth of human colon tumor. Oncogene 25: 480-486, 2006.

11. Chen CH, Lu PJ, Chen YC, Fu SL, Wu KJ, Tsou AP, Lee YC, Lin TC, Hsu SL, Lin WJ, et al: FLJ10540-elicited cell transformation is through the activation of PI3-kinase/AKT pathway. Oncogene 26: 4272-4283, 2007.

12. Inoda S, Hirohashi Y, Torigoe T, Nakatsugawa M, Kiriyama K, Nakazawa E, Harada K, Takasu H, Tamura Y, Kamiguchi K, et al: Cep55/c10orf3, a tumor antigen derived from a centrosome residing protein in breast carcinoma. J Immunother 32: 474-485, 2009.

13. Chen $\mathrm{CH}$, Chien CY, Huang CC, Hwang CF, Chuang HC, Fang FM, Huang HY, Chen CM, Liu HL and Huang CY: Expression of FLJ10540 is correlated with aggressiveness of ora cavity squamous cell carcinoma by stimulating cell migration and invasion through increased FOXM1 and MMP-2 activity. Oncogene 28: 2723-2737, 2009.

14. Chen CH, Lai JM, Chou TY, Chen CY, Su LJ, Lee YC, Cheng TS, Hong YR, Chou CK, Whang-Peng J, et al: VEGFA up-regulates FLJ10540 and modulates migration and invasion of lung cancer via PI3K/AKT pathway. PLoS One 4: e5052, 2009.

15. Chang YC, Chen YJ, Wu CH, Wu YC, Yen TC and Ouyang P: Characterization of centrosomal proteins Cep55 and pericentrin in intercellular bridges of mouse testes. J Cell Biochem 109: $1274-1285,2010$

16. Tao J, Zhi X, Tian Y, Li Z, Zhu Y, Wang W, Xie K, Tang J, Zhang X, Wang L and Xu Z: CEP55 contributes to human gastric carcinoma by regulating cell proliferation. Tumour Biol 35 4389-4399, 2014.

17. Talsma K, van Hagen P, Grotenhuis BA, Steyerberg EW, Tilanus HW, van Lanschot JJ and Wijnhoven BPL: Comparison of the 6th and 7th Editions of the UICC-AJCC TNM Classification for Esophageal Cancer. Ann Surg Oncol 19: 2142-2148, 2012.

18. Schmittgen TD and Livak KJ: Analyzing real-time PCR data by the comparative C(T) method. Nat Protoc 3: 1101-1108, 2008.

19. Dresner SM and Griffin SM: Pattern of recurrence following radical oesophagectomy with two-field lymphadenectomy. Br J Surg 87: 1426-1433, 2000.

20. Tanabe G, Baba M, Kuroshima K, Natugoe S, Yoshinaka H, Aikou T and Kajisa T: Clinical evaluation of the esophageal lymph flow system based on RI uptake of dissected regional lymph nodes following lymphoscintigraphy. Nihon Geka Gakkai Zasshi 87: 315-323, 1986 (In Japanese)
21. Chen G, Wang Z, Liu XY, Zhang MY and Liu FY: Abdominal lymph node metastasis in patients with mid thoracic esophageal squamous cell carcinoma. World J Surg 33: 278-283, 2009.

22. Li B, Chen H, Xiang J, Zhang Y, Li C, Hu H and Zhang Y: Pattern of lymphatic spread in thoracic esophageal squamous cell carcinoma: A single-institution experience. J Thorac Cardiovasc Surg 144: 778-785; discussion 785-786, 2012.

23. Gu Y, Swisher SG, Ajani JA, Correa AM, Hofstetter WL, Liao Z, Komaki RR, Rashid A, Hamilton SR and Wu TT: The number of lymph nodes with metastasis predicts survival in patients with esophageal or esophagogastric junction adenocarcinoma who receive preoperative chemoradiation. Cancer 106: 1017-1025, 2006.

24. Natsugoe S, Yoshinaka H, Shimada M, Sakamoto F, Morinaga T, Nakano S, Kusano C, Baba M, Takao S and Aikou T: Number of lymph node metastases determined by presurgical ultrasound and endoscopic ultrasound is related to prognosis in patients with esophageal carcinoma. Ann Surg 234: 613-618, 2001.

25. Baba M, Aikou T, Natsugoe S, Kusano C, Shimada M, Nakano S, Fukumoto T and Yoshinaka H: Quality of life following esophagectomy with three-field lymphadenectomy for carcinoma, focusing on its relationship to vocal cord palsy. Dis Esophagus 11: 28-34, 1998.

26. Matsubara T, Ueda M, Takahashi T, Nakajima T and Nishi M: Localization of recurrent disease after extended lymph node dissection for carcinoma of the thoracic esophagus. J Am Coll Surg 182: 340-346, 1996.

27. Yu Y, Wang Z, Liu XY, Zhu XF and Chen QF: Therapeutic efficacy comparison of two surgical procedures to treat middle thoracic esophageal carcinoma. World J Surg 34: 272-276, 2010.

28. Chen H, Wang Z, Yang Z, Shang B, Liu X and Chen G Prospective study of adjuvant radiotherapy on preventing lymph node metastasis after Ivor-lewis esophagectomy in esophageal cancer. Ann Surg Oncol 20: 2721-2726, 2013.

29. Gromley A, Yeaman C, Rosa J, Redick S, Chen CT, Mirabelle S, Guha M, Sillibourne J and Doxsey SJ: Centriolin anchoring of exocyst and SNARE complexes at the midbody is required for secretory-vesicle-mediated abscission. Cell 123: 75-87, 2005.

30. Fujiwara T, Bandi M, Nitta M, Ivanova EV, Bronson RT and Pellman D: Cytokinesis failure generating tetraploids promotes tumorigenesis in p53-null cells. Nature 437: 1043-1047, 2005.

31. Glotzer M: The molecular requirements for cytokinesis. Science 307: 1735-1739, 2005.

32. Paweletz N: Walther flemming: Pioneer of mitosis research. Nat Rev Mol Cell Biol 2: 72-75, 2001.

33. Carlton JG and Martin-Serrano J: Parallels between cytokinesis and retroviral budding: A role for the ESCRT machinery. Science 316: 1908-1912, 2007.

34. Sakai M, Shimokawa T, Kobayashi T, Matsushima S, Yamada Y, Nakamura Y and Furukawa Y: Elevated expression of C10orf3 (chromosome 10 open reading frame 3 ) is involved in the growth of human colon tumor. Oncogene 25: 480-486, 2006. 